\title{
Estrogen enhances tumor growth and angiogenesis indirectly via mediation of bone marrow-derived cells as well as directly through stimulation of tumor and endothelial cells
}

\author{
YINGCHEN ZHUO ${ }^{1}$, XUEQIAN LI $^{1}$, QIAOWEI ZHENG ${ }^{1}$, XINGJUN FAN $^{2}$, WENBING MA $^{1}$, \\ JINGGUO CHEN $^{1}$, XUE ZHAO ${ }^{1}$, PEIPEI ZHAO ${ }^{1}$, XUANLIN LIU ${ }^{1}$, \\ FENGRU TANG $^{1}$, KAI CHENG ${ }^{1}$ and WEIYI FENG ${ }^{1}$ \\ ${ }^{1}$ Department of Pharmacy, The First Affiliated Hospital of Xi'an Jiaotong University, Xi'an, Shaanxi 710061, P.R. China;
${ }^{2}$ Department of Pathology, Case Western Reserve University, Cleveland, OH 4410, USA
}

Received July 11, 2017; Accepted July 13, 2018

DOI: $10.3892 /$ or.2018.6631

\begin{abstract}
Estradiol (E2) is a prime culprit for enhancing the progression of female hormone-related cancers. Bone marrow-derived cells (BMDCs) have been found to play a pivotal role in tumor growth. Estrogen receptors (ERs) are also found on certain subtypes of BMDCs, in addition to endothelial cells (ECs) and certain tumor cells. However, the role of BMDCs in E2-induced tumor biology is still unclear. Thus, the effects of E2 on ER-negative 4T1 breast cancer growth, the mobilization and recruitment of BMDCs, and interactions among BMDCs, ECs, and 4T1 cells were investigated. The results showed that E2 potentiated 4T1 tumor growth and angiogenesis in mice subjected to sham operation, ovariectomy (OVX), or OVX and E2 replacement treatment. E2 supplementation in mice with OVX upregulated the transcription of stromal cell-derived factor-1 (SDF-1) mRNA in tumor tissues and enhanced the recruitment of BMDCs into tumor tissues in vivo. E2 deficiency significantly decreased proangiogenic CXCR $4^{+}, \mathrm{\beta}_{3}{ }^{+}, \mathrm{Sca}-1^{+}$ and $\mathrm{CXCR} 4^{+}{\beta_{3}}^{+} \mathrm{BMDC}$ circulating in the peripheral blood. Cell-based system analyses showed that E2 augmented the
\end{abstract}

Correspondence to: Professor Weiyi Feng, Department of Pharmacy, The First Affiliated Hospital of Xi'an Jiaotong University, 277 Yanta West Road, Xi'an, Shaanxi 710061, P.R. China

E-mail: fengweiyi@mail.xjtu.edu.cn

Abbreviations: BMDCs, bone marrow-derived cells; BMT, bone marrow transplantation; CM, conditioned media; E2, 17 $\beta$-estradiol; E2V, estradiol valerate; ECs, endothelial cells; ERs, estrogen receptors; H\&E, hematoxylin and eosin; HUVECs, human umbilical vein endothelial cells; MTT, 3-(4,5-dimethylthiazol-2-yl)-2, 5-diphenyltetrazolium bromide; MVA, microvascular area; OD, optical density; OVX, ovariectomy; SDF-1, stromal cell-derived factor-1; SO, sham operation; WBCs, white blood cells

Key words: angiogenesis, breast carcinoma, bone marrow cells, carcinogenesis, estradiol transcription of $\beta_{3}$ mRNA in ECs, increased the adhesion of BMDCs to ECs. In addition, E2 enhanced the BMDC-induced EC proliferation and migration, the BMDC-induced 4T1 proliferation and the 4T1-stimulated EC proliferation in addition to enhancing the proliferation of tumor cells and the migration of ECs in vitro. Therefore, E2 enhanced the growth of breast tumors by stimulating tumor cells and ECs directly, as well as by increasing proangiogenic BMDC mobilization and recruitment leading to augmentation of the tumor and EC functions indirectly by cell proliferation assay. These findings reveal a separate mechanism via which E2 promotes the growth of female hormone-dependent tumors, which may be useful in explorations of new therapies for related cancers.

\section{Introduction}

Estrogen regulates the function of many cell types, mostly by binding its receptors (1). Estrogen receptors (ERs) influence many physiological processes in mammals (2-5). Two classes of ERs exist: I) Nuclear ERs, which are ligand-regulated transcription factors that activate the expression of target genes in a large variety of responses $(6,7)$, and II) membrane ERs, which are mostly G-protein-coupled receptors that mediate nongenomic actions $(8,9)$. Despite the beneficial actions of endogenous estrogen under physiological conditions and its preventative effects in certain cancers, estrogen also exerts deleterious exacerbating effects in other disease states (10). There is considerable evidence that circulating estrogens are positively associated with the risk of breast cancer, accounting for almost $25 \%$ of cancer cases in females $(11,12)$. It is therefore not surprising that there is a wealth of data demonstrating estrogen to be a key carcinogen for these malignancies (13).

The direct effect of estrogen on tumor cells and endothelial cells (ECs) has been proposed as a major factor in stimulating tumor growth. On the one hand, estrogen regulates the proliferation, migration and adhesion of tumor cells, while on the other hand, blood vessel formation is also closely related to the estrogen level. The vascular growth in the uterus is routinely observed to be associated with fluctuating estrogen levels under physiological conditions (14). Under pathological 
conditions, estrogen is the key regulatory factor in tumor angiogenesis (15). As a result, activation of the ERs on tumor cells and ECs is widely considered to be the main mechanism underlying how estrogen enhances tumor growth.

There are clear signs that most immune cells express ERs, suggesting that these cells, which are mostly derived from bone marrow, are sensitive to estrogen (16). There is evidence that bone marrow-derived cells (BMDCs) recruited from the circulating blood in local angiogenic tissues play a more important role than local ECs in tumor angiogenesis (17). Although constituting a minority of the total stromal cell population in a tumor, BMDCs play a key role in tumor progression (18). Depletion of BMDCs in tumor tissues was found to result in delayed tumorigenesis (18). BMDCs are heterogeneous cells derived from the bone marrow (19), and certain subtypes of BMDCs [e.g., endothelial progenitor cells (EPCs)] have been found necessary to mediate estrogen-induced proangiogenesis in animal models $(20,21)$. In addition, BMDC-associated ERs were found to be the main target for estrogen to activate EPCs that were subsequently involved in tubulogenesis directly or enhance neovascularization by secreting angiogenic cytokines indirectly $(20,21)$. In addition, estrogen markedly enhances the genesis of breast cancers that lack ER expression or express ERs in very few cells $(16,22)$. This phenomenon implies that the effect of E2 on the growth of breast cancer is dependent not only on the local tumor cells but also on the systematic environment of the tumor cells. However, the exact mechanisms underlying how estrogen enhances tumor progression are still unclear.

Estradiol (E2) is the predominant estrogen. This study used E2 and ER-negative breast cancer 4T1 cells to investigate how estrogen regulates tumor growth. Roles of BMDCs in estrogen-induced tumor growth were defined. Understanding these mechanisms is vital for providing a theoretical basis for clinical E2-induced cancer therapies.

\section{Materials and methods}

Cell culture. Mouse mammary tumor cell line 4T1 and human umbilical vein endothelial cells (HUVECs) were kind gifts from the Cancer Research Center of Xi'an Jiao Tong University. Cells were cultured in RPMI-1640 medium supplemented with $10 \% \mathrm{FBS}$, penicillin 10,000 IU/ml and streptomycin $10,000 \mu \mathrm{g} / \mathrm{ml}$. All cell lines were grown at $37^{\circ} \mathrm{C}$ and $5 \% \mathrm{CO}_{2}$.

Animal model and in vivo tumorigenesis experiments (23). All animal procedures were conducted in accordance with the protocol approved by the Xi'an Jiaotong University Animal Care and Use Committee and housed under constant temperature, humidity, and lighting (12-h light per day) and allowed free access to food and water. Female BALB/c mice (aged, 6-8 weeks) were subjected to ovariectomy (OVX) or a sham operation (SO). The OVX mice were randomized into four groups ( $n=7$ in each group): One of the OVX groups received normal saline as a control (referred to as 'control' below), while the remaining three groups were gavaged with estradiol valerate (E2V, Bayer, Germany) at a doses of $0.27,0.8$, and $2.4 \mathrm{mg} / \mathrm{kg} / \mathrm{day}$.

For tumorigenesis experiments, tumors were induced by implanting $6 \times 10^{5} 4 \mathrm{~T} 1$ cells subcutaneously (16).
4T1-tumor-bearing mice were treated intragastrically with normal saline or different doses of E2V continuously. Tumor diameters were measured on days 6,9 and 12, and the tumor growth curve in each group was recorded. The maximum $(L)$ and minimum $(W)$ axes of the tumor were measured, with the tumor volume calculated as $0.5 L W^{2}$. After 16 days, mice were euthanized by cervical dislocation after anesthetization and the tumor tissues were isolated.

Histology and immunohistochemistry. The tissue sections were stained immunohistochemically or with hematoxylin and eosin (H\&E). The immunohistochemistry process was performed with CD31 (1:50 dilution; cat. no. ab28364; Abcam, Cambridge, UK) as the primary antibody in accordance with the instructions provided with the Histostain-Plus kit (4Abio, Beijing, China).

Cell proliferation assay. 17- $\beta$ E2 (Sigma; Merck KGaA, Darmstadt, Germany) was solubilized in absolute ethanol. Since BMDCs are mobilized into the blood circulation and recruited to tumor tissue (24), circulating white blood cells (WBCs) isolated using red blood cell (RBC) lysis buffer were used as a substitute for BMDCs in the in vitro experiments. WBCs ( $8 \times 10^{5}$ each group) were treated with RPMI-1640 medium containing 17- $\beta$ E2 at several different doses for $72 \mathrm{~h}$ at $37^{\circ} \mathrm{C}$. Thereafter, the supernatants were isolated by centrifugation and used as conditioned media (CM).

ECs or $4 \mathrm{~T} 1$ cells $\left(3 \times 10^{3}\right.$ cells/well $)$ were plated onto a 96-well plate and cultured with normal medium for $24 \mathrm{~h}$, and then replaced with E2-containing medium or CM $(200 \mu \mathrm{l})$ for $48 \mathrm{~h}$. E2-containing medium or CM was replaced with MTT (5 $\mu \mathrm{g} / \mathrm{ml}$, Beyotime Institute of Biotechnology, Shanghai, China), and cells were incubated for another $4 \mathrm{~h}$. The medium was then discarded and DMSO was added. The 96-well plate was shaken for $10 \mathrm{~min}$ to dissolve the formazan crystals. The absorbance (OD value) was measured at $490 \mathrm{~nm}$ using a microplate reader (Bio-Rad 550; Bio-Rad Laboratories, Inc., Hercules, CA, USA). Cultures in normal medium alone were used as negative controls. The effects of E2 and/or BMDCs on ECs or 4T1 cell proliferation were expressed as the 'added value' of OD: Added value $=(O D$ value in the experimental group)-(average OD value in the control group).

The cell proliferation and migration was compared between E2-free and E2-treated WBC groups using the following four experimental groups: WBC/0+0 (E2-free WBC CM was mixed with the same volume of E2-free medium), $\mathrm{WBC} / 1+1$ (1-nM E2-treated WBC CM was mixed with the same volume of 1-nM E2-containing medium), WBC/0+1 (E2-free WBC $\mathrm{CM}$ was mixed with the same volume of 1-nM E2-containing medium), and WBC/1+0 (1-nM E2-treated WBC CM was mixed with the same volume of E2-free medium). Similarly, the cell proliferation was compared between E2-free and E2-treated 4T1 cell groups using another four experimental groups: 4T1/0+0, 4T1/1+1, 4T1/0+1, and 4T1/1+0.

In vitro scratch assay. HUVECs $\left(8 \times 10^{5}\right)$ were cultured to confluence on 24-well plates (Corning Costar, Corning, NY, USA). Confluent cells were scraped with a $200 \mu$ l pipette tip and then incubated with WBC CM (as is shown in Cell proliferation 
Table I. Primer sequences for RT-PCR.

\begin{tabular}{llc}
\hline Primers & \multicolumn{1}{c}{ Sequences } & Product size (bp) \\
\hline Mouse SDF-1 (F) & 5'-AGCCAACGTCAAGCATCTG-3' & 263 \\
Mouse SDF-1 (R) & 5'-TAATTTCGGGTCAATGCACA-3' & 106 \\
Mouse $\beta$-actin (F) & 5'-GAGACCTTCAACACCCCAGC-3' \\
Mouse $\beta$-actin (R) & 5'-ATGTCACGCACGATTTCCC-3' & 205 \\
Human $\beta 3(\mathrm{~F})$ & 5'-GCCAGCACCATCTCTTTACC-3' & 112 \\
Human $\beta 3(\mathrm{R})$ & 5'-GCACTCTCTCCCTTTGAGGA-3' & \\
Human $\beta$-actin (F) & 5'-TGACGTGGACATCCGCAAAG-3' & \\
\hline
\end{tabular}

F, forward; R, reverse.

assay). The scratch was photographed at 0,6 and $12 \mathrm{~h}$ and data was analysed using Image-Pro Plus (version 6.0).

Sex-mismatched bone-marrow transplantation (25). Recipient female mice were lethally irradiated at $8 \mathrm{~Gy}$. Bone marrow was harvested from the femurs and tibias of male donor mice. Total bone marrow was liberated using 20-gauge needles to produce a single-cell suspension in RPMI-1640 medium with $10 \%$ FBS. The bone-marrow cell suspensions were centrifuged at 1,000 rpm for $5 \mathrm{~min}$, and the obtained pellets were resuspended in sterile RPMI-1640 medium at $3 \times 10^{7}$ cells $/ \mathrm{ml}$. The bone marrow cells $\left(1 \times 10^{7}\right)$ were then injected into the lateral tail vein of a recipient female mouse, after which sulfamethoxazole solution was administered for 4 weeks. Tumor cell implantation was performed 8 weeks after bone marrow transplantation (BMT).

Fluorescence in situ hybridization. Tumor samples obtained from sex-mismatched BMT mice were embedded in optimal cutting temperature compound and stored at $-80^{\circ} \mathrm{C}$. Frozen tissues were sectioned and fixed in paraformaldehyde for $10 \mathrm{~min}$, and the frozen sections were rinsed with 0.01 M PBS for $10 \mathrm{~min}$. The Y chromosome was detected by Mouse SRY DNA FISH kit (TBD Science, Tianjin, China) following the manufacturer's instructions. The sections were finally counterstained with blue fluorescent 4'-6-diamidino-2-phenylindole (DAPI, Sigma; Merck KGaA). Images were captured using fluorescence microscopy (Olympus BX51; Olympus Corp., Tokyo, Japan).

Flow cytometry. Peripheral blood was collected from the mouse abdominal aorta and then treated with RBC lysis buffer. Leukocytes $\left(1 \times 10^{6}\right)$ were incubated with APC-anti-CXCR4 (1:100 dilution; cat. no. 558644; BD Pharmingen; BD Biosciences, Franklin Lakes, NJ, USA), PE-anti-CD61 (1:80 dilution; cat. no. 104307; Biolegend, Inc., San Diego, CA, USA), FITC-anti-Sca-1 (1:100 dilution; cat. no. 108115; Biolegend, Inc.), or AF488-anti-CD11b (1:200 dilution, cat. no. 101205; BioLegend, Inc.) antibodies following the appropriate protocol. Double staining was also performed with anti-CXCR4 and anti-CD61 antibodies. Labeled cells were fixed with paraformaldehyde. Flow cytometry on a FACScan (BD Biosciences) was used to analyze 10,000 events per sample.
$R T-P C R$. Total RNA was extracted using RNA fast 2000 kit (Fastagen, Shanghai, China) following the manufacturer's instructions. The cDNA was synthesized via Prime Script RT Master Mix Perfect Real-Time kit (DRR036A; Takara, Tokyo, Japan). The primer sequences used in this study along with the expected product sizes are listed in Table I. The cycling protocol for PCR involved incubating the samples at $94^{\circ} \mathrm{C}$ for 2 min followed by 35 cycles of denaturation at $94^{\circ} \mathrm{C}$ for $30 \mathrm{sec}$, annealing at $55^{\circ} \mathrm{C}$ for $30 \mathrm{sec}$, and extension at $72^{\circ} \mathrm{C}$ for $30 \mathrm{sec}$, with a final cycle of incubation at $72^{\circ} \mathrm{C}$ for $2 \mathrm{~min}$. The amplification products were analyzed by electrophoresis (Beijing Junyi, Beijing, China) in agarose gels and detected under UV illumination (Bio-Rad Laboratories) after staining with nucleic acid dye (DuRed; FanBo Biochemicals, Beijing, China). Images were analyzed using a quantitative analysis system (Quantity One Analysis Software, version 4.6.2; Bio-Rad Laboratories).

Adhesion assay. HUVECs were cultured to confluence on 12 -well plates precoated with gelatin, and then treated with $17-\beta$ E2. After $24 \mathrm{~h}$, the HUVEC monolayers were washed three times with PBS. Calcein-AM-labeled human WBC $\left(1 \times 10^{5}\right)$ suspensions were added to each well and incubated for $60 \mathrm{~min}$ at $37^{\circ} \mathrm{C}$. The nonadherent calcein-AM-labeled WBCs were then removed. Adherent human WBCs were observed using fluorescence microscopy (Leica Microsystems, Wetzlar, Germany), with the fluorescence intensity quantified using a fluorescence analyzer (excitation filter $495 \mathrm{~nm}$, emission filter $515 \mathrm{~nm}$ ) (PerkinElmer, Inc., Waltham, MA, USA).

Statistical analysis. Data are represented as the mean \pm SEM and were analyzed by a two-tailed Student t-test or one-way analysis of variance (ANOVA) using GraphPad Prism version 4.03 software (GraphPad Software, Inc., La Jolla, CA, USA). P-values of 0.05 or less were regarded as statistically significant.

\section{Results}

E2 enhances murine $4 T 1$ breast cancer growth and angiogenesis in vivo. $4 \mathrm{~T} 1$ cells were implanted subcutaneously into OVX female mice, and tumor volume were measured every 3 days (Fig. 1A). At 16 days after implantation, 4T1 tumor growth in 
A

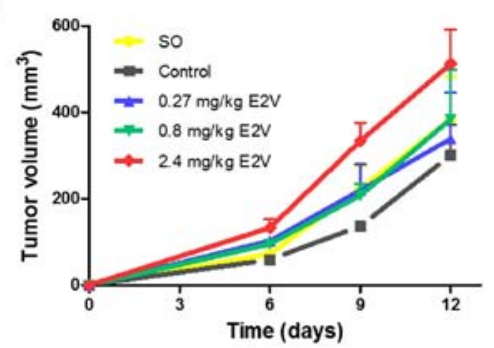

C

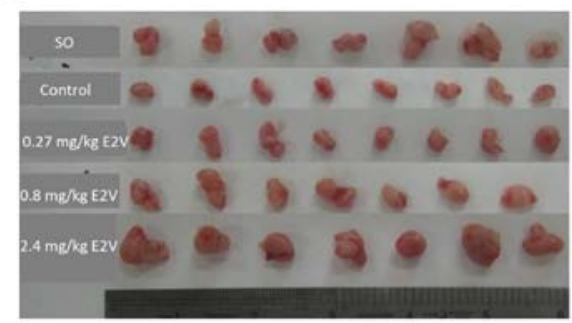

E
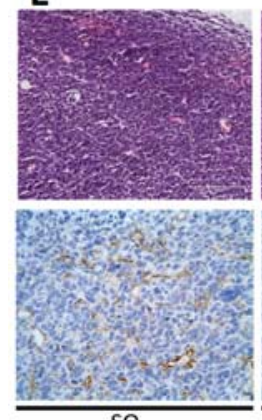
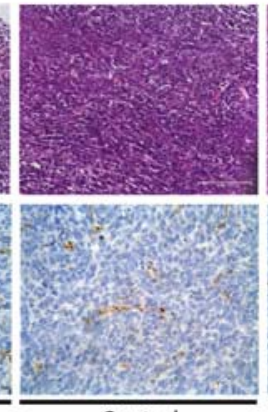

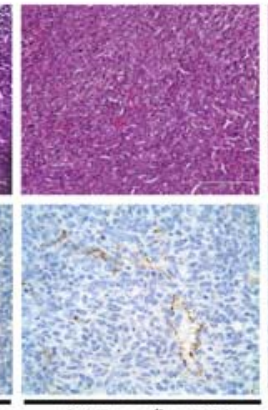

$0.27 \mathrm{mg} / \mathrm{kg} \mathrm{E2V}$
B

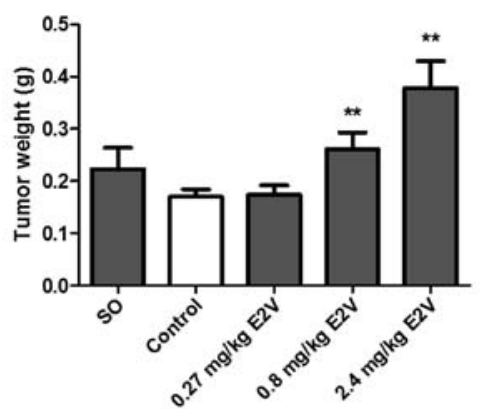

D

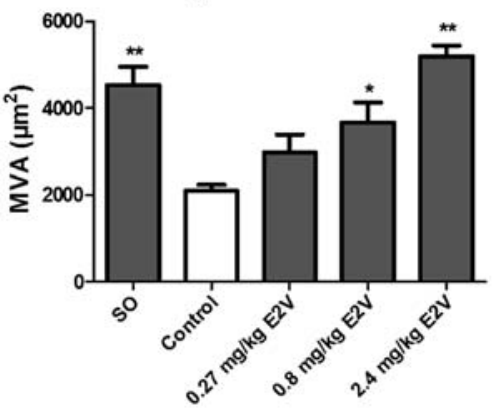

Figure 1. Increased ER-negative tumor growth in vivo and vascularization following treatment with E2. In vivo growth curves (A) and weights (B and C) of 4T1 tumors from SO mice, OVX mice (control), and OVX mice with 0.27, 0.8, and $2.4 \mathrm{mg} / \mathrm{kg}$ E2V replacement, $\mathrm{n}=7$ in each group. (D and E) H\&E (scale bars, $145 \mu \mathrm{m}$ ) or anti-CD31-stained tumor tissue sections (scale bars, $50 \mu \mathrm{m}$ ) and quantification of the CD31 ${ }^{+} \mathrm{MVA}$. Tumor weights and MVA are indicated as mean and SEM values. ${ }^{*} \mathrm{P}<0.05,{ }^{* *} \mathrm{P}<0.01$ vs. control. ER, estrogen receptor; SO, sham operation; OVX, ovariectomy; H\&E, hematoxylin and eosin; MVA, microvascular area; E2V, estradiol valerate.
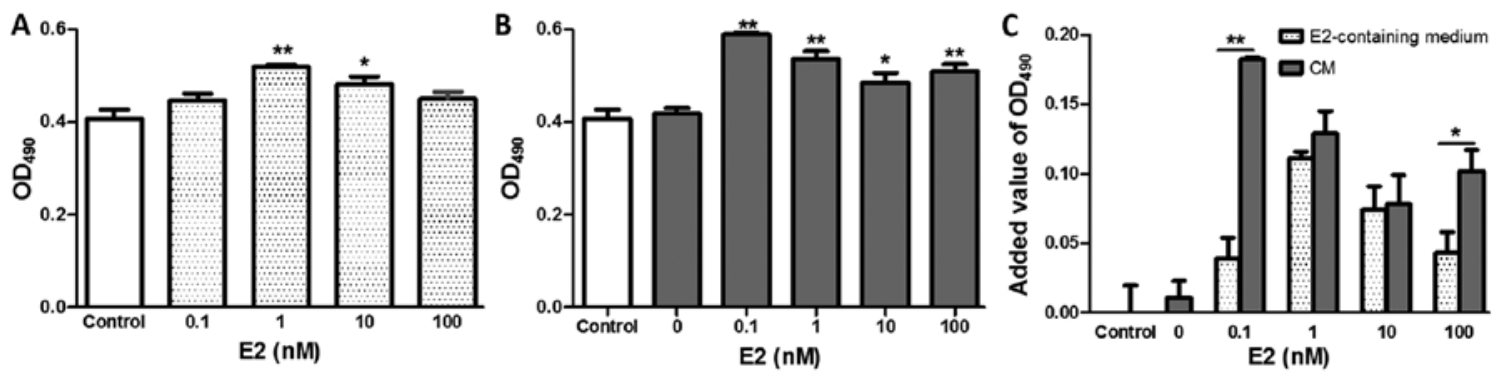

Figure 2. Effects of E2 and E2-treated BMDCs on the proliferation of $4 \mathrm{~T} 1$ cells with E2 at concentrations of 0.1, 1, 10, and $100 \mathrm{nM}$, respectively. (A) Proliferation of E2-treated 4T1 cells after $48 \mathrm{~h}$ of culture measured with the MTT assay. (B) Proliferation of 4T1 cells after treatment with E2-stimulated BMDC CM. (C) Comparison of 4T1 cell proliferation between groups treated with E2 and with CM from BMDCs stimulated with E2. Data are mean and SEM values. ${ }^{*} \mathrm{P}<0.05,{ }^{* *} \mathrm{P}<0.01$ vs. the control or E2-free BMDC CM group. BMDCs, bone marrow-derived cells; E2, 17ß-estradiol; MTT, 3-(4,5-dimethylthiazol-2-yl)-2, 5-diphenyltetrazolium bromide; CM, conditioned media.

the OVX mice did not differ significantly from that in SO mice. As shown in Fig. 1B and C, treatment with 0.8 and $2.4 \mathrm{mg} / \mathrm{kg}$ E2V led to significant increases in tumor weights, compared with the placebo-treated control group $(\mathrm{P}<0.01)$.

To observe the effect of E2 on tumor angiogenesis in ER-negative 4T1 cell-derived tumors, tumor tissues were stained with H\&E staining and immunohistochemistry was conducted (Fig. 1E). Quantification of tumor CD31+ vessels revealed that the microvascular area (MVA) field of view was notably larger in the 0.8 and $2.4 \mathrm{mg} / \mathrm{kg}$ E2V groups than in the OVX group $(\mathrm{P}<0.05$ and $\mathrm{P}<0.01$, respectively) (Fig. 1D).

E2 and the CM of E2-treated BMDCs enhances the proliferation of $4 \mathrm{T1}$ cells. E2 affected the proliferation of 4T1 

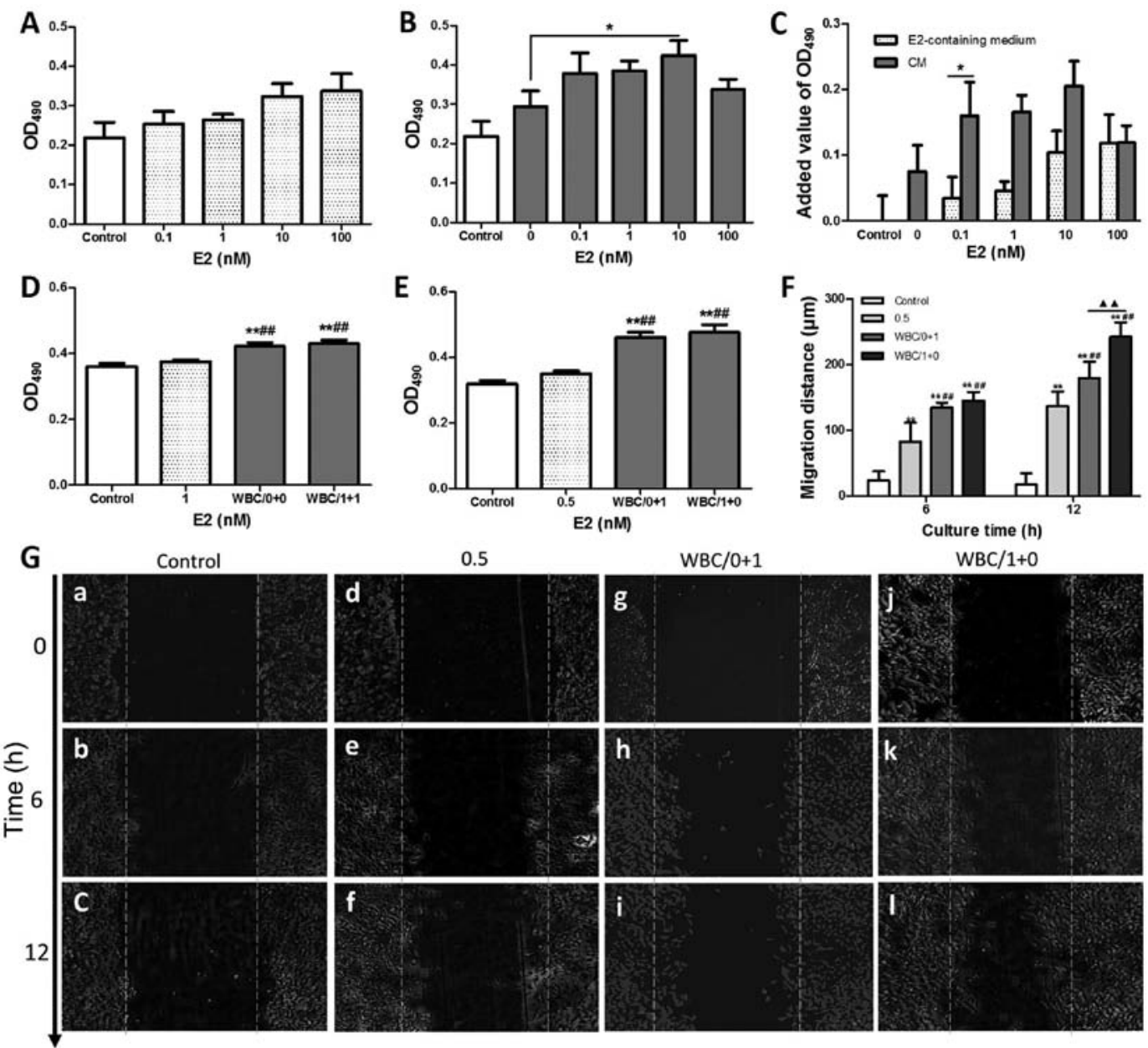

Figure 3. Effects of E2 and E2-treated BMDCs on the proliferation and migration of ECs. (A and B) MTT assay of EC proliferation after treatment with E2 or E2-stimulated BMDC CM for E2 at concentrations of 0.1, 1, 10 and $100 \mathrm{nM}$, respectively. (C) Comparison of EC proliferation between groups treated with E2, and with CM from BMDCs stimulated with E2. (D) MTT assay of HUVEC proliferation after $48 \mathrm{~h}$ of treatment with E2-containing medium, E2-free WBC CM or E2-stimulated WBC CM. (E) Comparison of HUVEC proliferation between groups treated with CM from WBCs stimulated with or without E2. ( $\mathrm{F}$ and $\mathrm{G})$ In vitro scratch assay of HUVEC migration. Data are the mean and SEM values. ${ }^{* *} \mathrm{P}<0.01 \mathrm{vs}$. the control; ${ }^{* \#} \mathrm{P}<0.01 \mathrm{vs}$. the E2-containing medium groups; ${ }^{\Delta} \mathrm{P}<0.01, \mathrm{WBC} / 0+1$ group vs. $\mathrm{WBC} / 1+0$ group. E2, $17 \beta$-estradiol; BMDCs, bone marrow-derived cells; ECs, endothelial cells; MTT, 3-(4,5-dimethylthiazol-2-yl)-2, 5-diphenyltetrazolium bromide; CM, conditioned media; HUVECs, human umbilical vein endothelial cells; WBC, white blood cell; OD, optical density; WBC/0+0 (E2-free WBC CM was mixed with the same volume of E2-free medium); WBC/1+1 (1-nM E2-treated WBC CM was mixed with the same volume of 1-nM E2-containing medium); WBC/0+1 (E2-free WBC CM was mixed with the same volume of 1-nM E2-containing medium) and $\mathrm{WBC} / 1+0$ (1-nM E2-treated WBC CM was mixed with the same volume of E2-free medium).

cells in the concentration range from 0.1 to $100 \mathrm{nM}$ in vitro. The results showed that 1 or $10 \mathrm{nM}$ E2 significantly increased the proliferation of $4 \mathrm{~T} 1$ cells compared with the control (Fig. $2 \mathrm{~A}, \mathrm{P}<0.01$ or $\mathrm{P}<0.05$, respectively).

BMDCs are mobilized into the blood circulation and recruited to tumor tissue to enhance tumor angiogenesis and growth. To investigate the role of BMDCs in the E2 enhancement of tumor growth in vitro, the effects of E2-treated WBC $\mathrm{CM}$ on $4 \mathrm{~T} 1$ cell proliferation were observed. As shown in Fig. 2B, the 4T1 cell proliferation in E2-stimulated WBC CM groups was significantly increased by 40.9, 28.2, 16.0 and $21.8 \%$ for $\mathrm{E} 2$ at concentrations of $0.1,1,10$ and $100 \mathrm{nM}$, respectively, as compared with the E2-free WBC CM $(\mathrm{P}<0.01$, $\mathrm{P}<0.01, \mathrm{P}<0.05, \mathrm{P}<0.01$, respectively). The added value for $\mathrm{OD}$ in the E2-stimulated WBC CM groups for 0.1 or $100 \mathrm{nM} \mathrm{E2}$ was significantly higher than that at the same concentration of E2-containing medium groups, as well as that in the E2-free WBC CM group (Fig. 2C; $\mathrm{P}<0.01, \mathrm{P}<0.05$, respectively). These results suggest that $\mathrm{E} 2$ and $\mathrm{BMDCs}$ exert a synergistic effect on enhancing the proliferation of 4T1 cells at a certain concentration.

E2 and the CM of E2-treated BMDCs facilitate the proliferation and migration of ECs. Stimulation with E2 led to small increases in EC proliferation in vitro (Fig. 3A). To investigate the role of BMDCs in E2-induced angiogenesis, the effects of E2-treated WBC CM on EC proliferation were observed. As shown in Fig. 3B, although E2-free WBCs CM enhanced EC proliferation by $34.2 \%$ relative to normal medium, E2-treated WBC CM with E2 at concentrations of $0.1,1,10$, and $100 \mathrm{nM}$ significantly enhanced EC proliferation (all $\mathrm{P}<0.05$ ). Meanwhile, 0.1 to $100 \mathrm{nM}$ E2-treated WBC CM increased EC proliferation compared with E2-free BMDC CM, with the enhancement reaching statistical significance in the $10 \mathrm{nM}$ group $(\mathrm{P}<0.05)$. As shown in Fig. 3C, the added values for WBC-mediated E2-induced EC proliferation were higher than those for the E2-free WBC-mediated EC group and the E2-stimulated EC group at E2 concentrations ranging from 

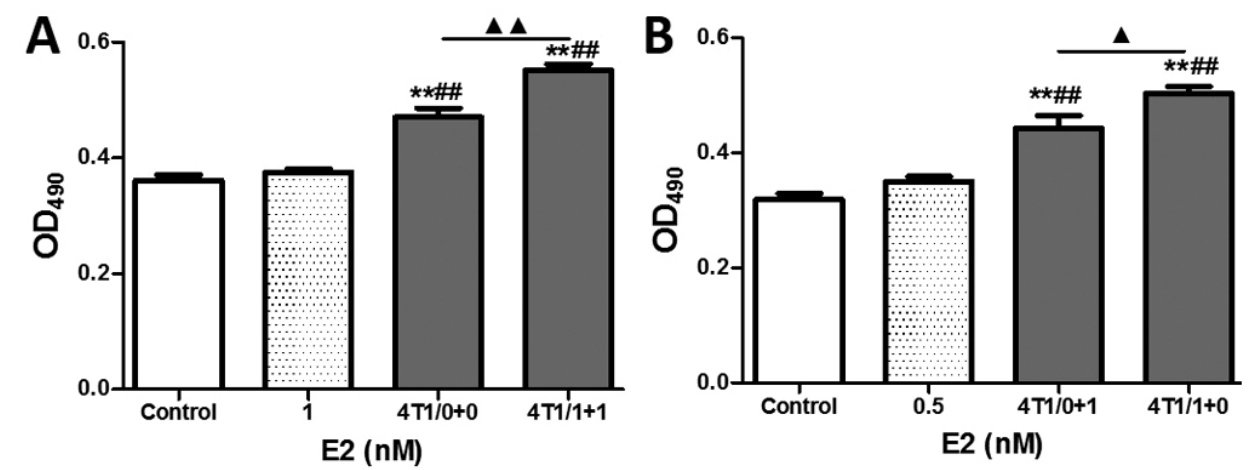

Figure 4. Effects of E2 and E2-treated 4T1 cells on the proliferation of HUVECs. (A) MTT assay of HUVEC proliferation after $48 \mathrm{~h}$ of treatment with E2-containing medium, E2-free 4T1 CM or E2-stimulated 4T1 CM. (B) Comparison of HUVEC proliferation between groups treated with CM from $4 \mathrm{~T} 1$ stimulated with or without E2. Data are mean and SEM values. ${ }^{* *} \mathrm{P}<0.01$ vs. the control; ${ }^{\# \#} \mathrm{P}<0.01$ vs. the E2-containing medium groups; ${ }^{\mathbf{A}} \mathrm{P}<0.05$, $4 \mathrm{~T} 1 / 0+1$ group vs. $4 \mathrm{~T} 1 / 1+0$ group; ${ }^{\mathbf{\Delta} \mathrm{P}}<0.01,4 \mathrm{~T} 1 / 0+0$ group vs. $4 \mathrm{~T} 1 / 1+1$ group. E2, $17 \beta$-estradiol; HUVECs, human umbilical vein endothelial cells; MTT, 3-(4,5-dimethylthiazol-2-yl)-2, 5-diphenyltetrazolium bromide; CM, conditioned media; 4T1/0+0 (E2-free 4T1 CM was mixed with the same volume of E2-free medium); 4T1/1+1 (1-nM E2-treated 4T1 CM was mixed with the same volume of 1-nM E2-containing medium); 4T1/0+1 (E2-free 4T1 CM was mixed with the same volume of 1-nM E2-containing medium) and 4T1/1+0 (1-nM E2-treated 4T1 CM was mixed with the same volume of E2-free medium.

0.1 to $10 \mathrm{nM}$. The differences in EC proliferation between the E2-stimulated EC groups and E2-treated WBC groups, as well as the differences between the E2-free WBC group and the E2-treated WBC groups, suggested a synergistic effect of E2 and BMDCs on EC proliferation, implying that the E2-induced EC proliferation was partly mediated by BMDCs at a certain concentration.

To investigate the synergistic action between E2 and BMDCs in enhancing HUVEC functions further, a more refined analysis was designed to observe HUVEC proliferation and migration with or without $1 \mathrm{nM} \mathrm{E2}$-treated WBC CM. As shown in Fig. 3D, although both E2-free WBC CM and E2-treated WBC CM significantly enhanced the proliferation of HUVECs in comparison to control or E2-treated ECs (all $\mathrm{P}<0.01$ ), no distinct increases were observed between the E2-free and E2-treated groups regardless of whether culturing was performed with either normal medium or $\mathrm{CM}$ for $48 \mathrm{~h}$.

Moreover, in order to eliminate the disturbance of HUVEC proliferation caused by the $\mathrm{E} 2$ concentrations in the medium, the final E2 concentration was adjusted to $0.5 \mathrm{nM}$ with $\mathrm{CM}$ and/or normal medium. A small difference was found between the $0.5 \mathrm{nM}$ E2 normal-media group and the control, indicating that E2 induced a slight increase in HUVEC proliferation. The marked enhancement of proliferation of HUVECs in groups of $\mathrm{WBC} / 0+1$ and $\mathrm{WBC} / 1+0$ compared with the $\mathrm{E} 2$ group indicated that BMDC CM significantly enhanced the effect of HUVECs on proliferation (all $\mathrm{P}<0.01$ ). However, no significant difference in HUVEC proliferation was found between the $\mathrm{WBC} / 0+1$ and $\mathrm{WBC} / 1+0$ groups, implying that the enhancement of HUVEC proliferation was associated with BMDC CM rather than with E2 (Fig. 3E).

In contrast, the migration of HUVECs increased markedly after 6 and $12 \mathrm{~h}$ of culture in the $0.5 \mathrm{nM}$ E2 group, WBC/0+1 group, and $\mathrm{WBC} / 1+0$ group, compared with the controls (Fig. $3 \mathrm{~F}$ and $\mathrm{G}$, all $\mathrm{P}<0.01$ ). Moreover, the medium containing WBC CM significantly enhanced HUVEC migration by 63.4 and $31.6 \%$ compared with $0.5 \mathrm{nM}$ E2 after 6 and $12 \mathrm{~h}$ of culture, respectively (all $\mathrm{P}<0.01$ ), while the medium containing E2-treated WBC CM (0.5 nM E2 in medium) markedly enhanced HUVEC migration compared with BMDC CM medium after $12 \mathrm{~h}$ of culture $(\mathrm{P}<0.01)$. These results indicate that BMDCs play an important role in mediating the enhancement of EC migration by E2.

E2-treated $4 T 1$ cells enhance the proliferation of HUVECs. As shown in Fig. 4A, although no obvious difference in HUVEC proliferation was found between the E2-treatment and control groups, both E2-free 4T1 CM and E2-treated 4T1 CM significantly enhanced HUVEC proliferation compared with the control and E2-treatment groups (all $\mathrm{P}<0.01$ ). To eliminate disturbances from differences in the E2 concentration in the medium, the final E2 concentration was adjusted to $0.5 \mathrm{nM}$ with $4 \mathrm{~T} 1 \mathrm{CM}$ and/or normal medium. The results showed that a significant increase of $13.5 \%$ was observed between the $4 \mathrm{~T} 1 / 0+1$ group and the $4 \mathrm{~T} 1 / 1+0$ group (Fig. $4 \mathrm{~B}$, $\mathrm{P}<0.05$ ), implying that $\mathrm{E} 2$ treatment markedly enhanced the HUVEC proliferation induced by $4 \mathrm{~T} 1$ cells. These findings suggest that the $\mathrm{E} 2$ enhancement of angiogenesis was partly mediated by $4 \mathrm{~T} 1$ tumor cells.

E2 induces the mobilization of $\mathrm{CXCR}^{+}, \beta_{3}{ }^{+}, \mathrm{Sca}-\mathrm{I}^{+}$and CXCR $4^{+} \beta_{3}{ }^{+}$BMDCs. The numbers of proangiogenic BMDCs in circulating blood, such as CXCR $4^{+}, \beta_{3}{ }^{+}$(also known as CD61), CXCR $4^{+} \beta_{3}{ }^{+}, \mathrm{CD} 11 \mathrm{~b}^{+}$, and Sca- $1^{+}$BMDCs, were assessed with flow cytometry. The results showed that CXCR $4{ }^{+}$BMDCs and $\beta_{3}{ }^{+}$BMDCs decreased sharply in mouse circulating blood after OVX (Fig. 5A and B). The CXCR4 ${ }^{+}$ BMDC counts were significantly rescued by 94.8 and $86.7 \%$ in mice after administering 0.27 and $0.8 \mathrm{mg} / \mathrm{kg}$ E2V, respectively, compared with control mice $(\mathrm{P}<0.01)$. The number of circulating $\beta_{3}{ }^{+}$BMDCs was markedly increased in the $2.4 \mathrm{mg} / \mathrm{kg}$ E2V group compared with the control group $(\mathrm{P}<0.01)$. The numbers of Sca- $1^{+}$BMDCs were notably increased by 35.3 and $22.2 \%$ in the 0.8 and $2.4 \mathrm{mg} / \mathrm{kg}$ E2V groups, respectively, compared with the control group (Fig. $5 \mathrm{C}, \mathrm{P}<0.01$ and $\mathrm{P}<0.05$ ). As shown in Fig. 5D, a slight increase in CD11b ${ }^{+}$BMDC counts in the $0.27 \mathrm{mg} / \mathrm{kg}$ group and mild decreases in CD11 $\mathrm{b}^{+}$ BMDC counts in the 0.8 and $2.4 \mathrm{mg} / \mathrm{kg}$ groups were observed in circulating blood following $\mathrm{E} 2 \mathrm{~V}$ treatment, but none of these results reached statistical significance. The counts of 

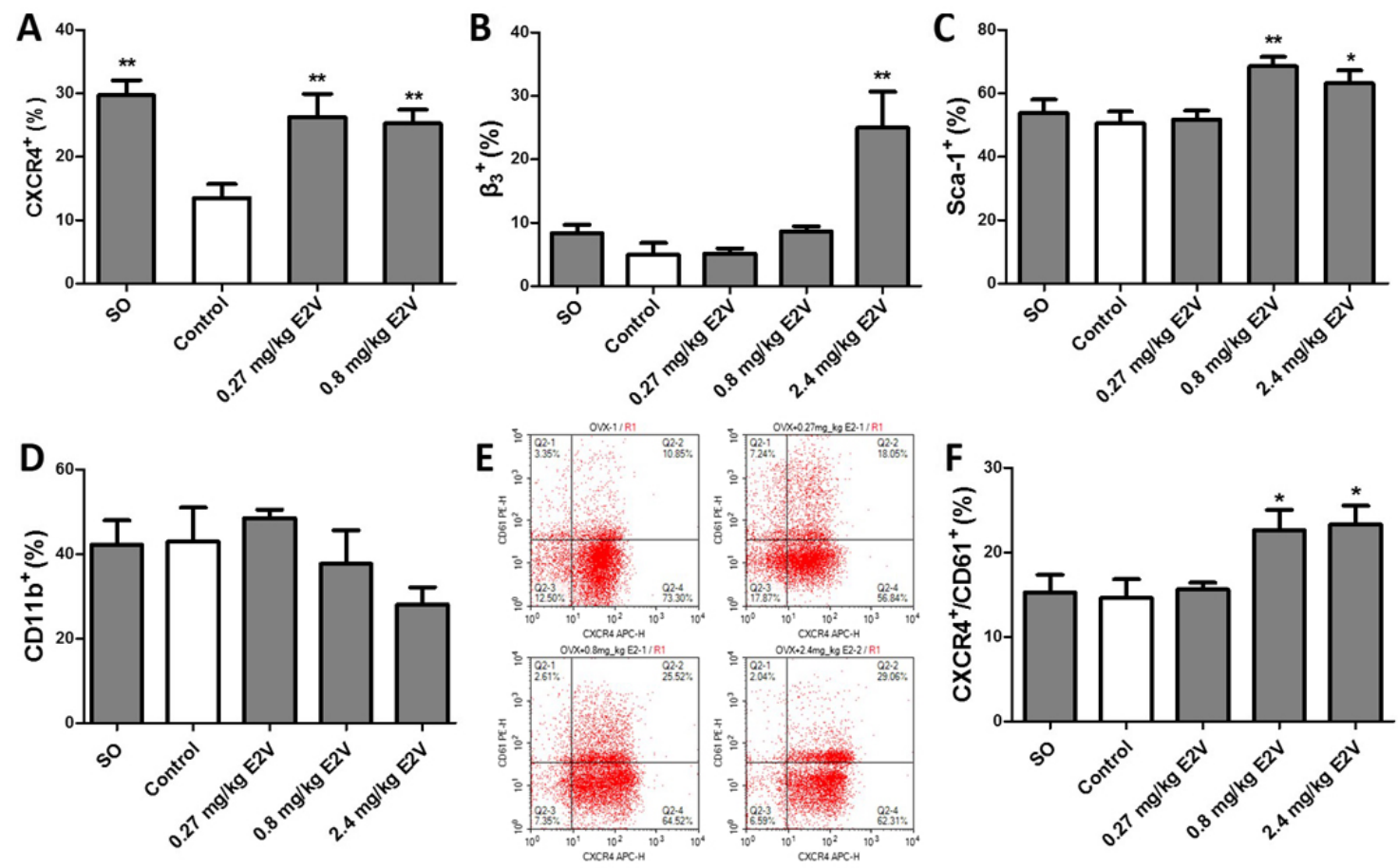

Figure 5. Effects of E2 on mobilization of CXCR4 $4^{+}, \beta_{3}{ }^{+}, \mathrm{Sca}-1^{+}$and CXCR4 ${ }^{+} \mathrm{CD} 61(\beta 3)^{+}$BMDCs in circulating blood. (A) Percentage of circulating CXCR4 ${ }^{+}$ cells in 4T1 tumor-bearing SO mice, control mice, and E2V-treated OVX mice $\left(0.27,0.8\right.$, and $2.4 \mathrm{mg} / \mathrm{kg}$ ). (B) Percentage of circulating $\beta 3^{+}$cells. (C) Percentage of circulating Sca- $1^{+}$cells. (D) Percentage of circulating CD11b ${ }^{+}$cells. (E and F) Percentage of circulating CXCR4 ${ }^{+} \mathrm{CD} 61^{+}$cells. Data are mean and SEM values. ${ }^{*} \mathrm{P}<0.05,{ }^{* *} \mathrm{P}<0.01$ vs. the control. E2, 17ß-estradiol; BMDCs, bone marrow-derived cells; SO, sham operation; OVX, ovariectomy; E2V, estradiol valerate.

circulating $\mathrm{CXCR} 4^{+} / \mathrm{CD} 61^{+}$BMDCs in the 0.8 and $2.4 \mathrm{mg} / \mathrm{kg}$ E2V groups were significantly increased, compared with the control group (Fig. 5E and $\mathrm{F}, \mathrm{P}<0.05$ ).

E2 enhances BMDC adhesion to ECs in vitro. The adhesion assay results showed that more BMDCs attached to an E2-pretreated EC monolayer than to an untreated EC monolayer, with the enhancement reaching statistical significance at $1,000 \mathrm{nM}$ (Fig. 6A, P<0.05). Furthermore, transcriptional expression of integrin $\beta_{3}$ mRNA was higher than that in the control following treatment of HUVECs with 1, 10, 100, and $1,000 \mathrm{nM} \mathrm{E} 2$, respectively, in vitro (Fig. $6 \mathrm{~B}, \mathrm{P}<0.01$ ), indicating that the enhanced adhesion of BMDCs to ECs was attributable to the stimulation of expression of integrin $\beta_{3}$ on ECs by E2.

E2 augments SDF-1 mRNA expression in tumor tissues and enhances the recruitment and retention of BMDCs in $4 T 1$ tumor tissues. As shown in Fig. 6C, the expression level of SDF-1 mRNA in tumor tissues was significantly higher in the 0.8 and $2.4 \mathrm{mg} / \mathrm{kg} \mathrm{E} 2 \mathrm{~V}$ groups than in the control group $(\mathrm{P}<0.01)$. To confirm that the recruitment of BMDCs in tumor tissues was related to $\mathrm{E} 2$, the retention of BMDCs in tumor tissues was analyzed in sex-mismatched BMT mouse models. The densities of Y-chromosome-positive cells in tumor tissues from the $0.27,0.8$, and $2.4 \mathrm{mg} / \mathrm{kg}$ E2V groups were increased by $141.4,251.7$ and $293.1 \%$, respectively, compared with the control group $(\mathrm{P}<0.05$, Fig. 6D).

\section{Discussion}

Evidence has been subsequently obtained of a central role of estrogen in the progression of breast cancers irrespective of whether they are positive or negative for ERs (16). These results imply that estrogen might provoke a systemic host response to induce tumor progression not only via tumor cells themselves in vivo.

There are multiple lines of evidence that estrogen directly modulates angiogenesis via ECs (14). The present experiments demonstrated that E2 enhanced murine ER-negative 4T1 tumor growth and angiogenesis in a dose-dependent manner. Nevertheless, BMDCs play a pivotal role in stimulating progression in tumors (26). ERs were found to be expressed in multiple BMDCs (16), and thus BMDCs might be involved in the development of E2-induced breast tumors.

These observations prompted the present study to observe interactions among BMDCs, ECs, and 4T1 tumor cells, as well as the effects of E2 on this process. Meanwhile, considering that tissue-resident BMDCs are recruited from circulating blood, WBCs were employed to observe the role of BMDCs in tumor progression and angiogenesis in the experiments. We found that E2 significantly enhanced ER-negative 4T1 tumor cell proliferation and that CM from E2-treated BMDCs-but not BMDC CM-enhanced the E2-induced enhancement of 4T1 tumor cell proliferation at certain concentrations. As shown in Fig. 2A and B, the greatest values of OD were observed at $1 \mathrm{nM}$ E2-containing medium group and at $0.1 \mathrm{nM}$ E2-treated WBC CM group respectively, which were not in sync. Meanwhile, the data in both (E2 and CM) mediums did not show linear dose-response dependence relationships. As a result, the increases of OD value between the E2 and E2-containing condition medium groups showed significance at the concentrations of 0.1 and $100 \mathrm{nM}$, but not at 1 and $10 \mathrm{nM}$. Moreover, although E2 enhanced the EC proliferation only slightly, BMDCs enhanced E2-induced EC proliferation 

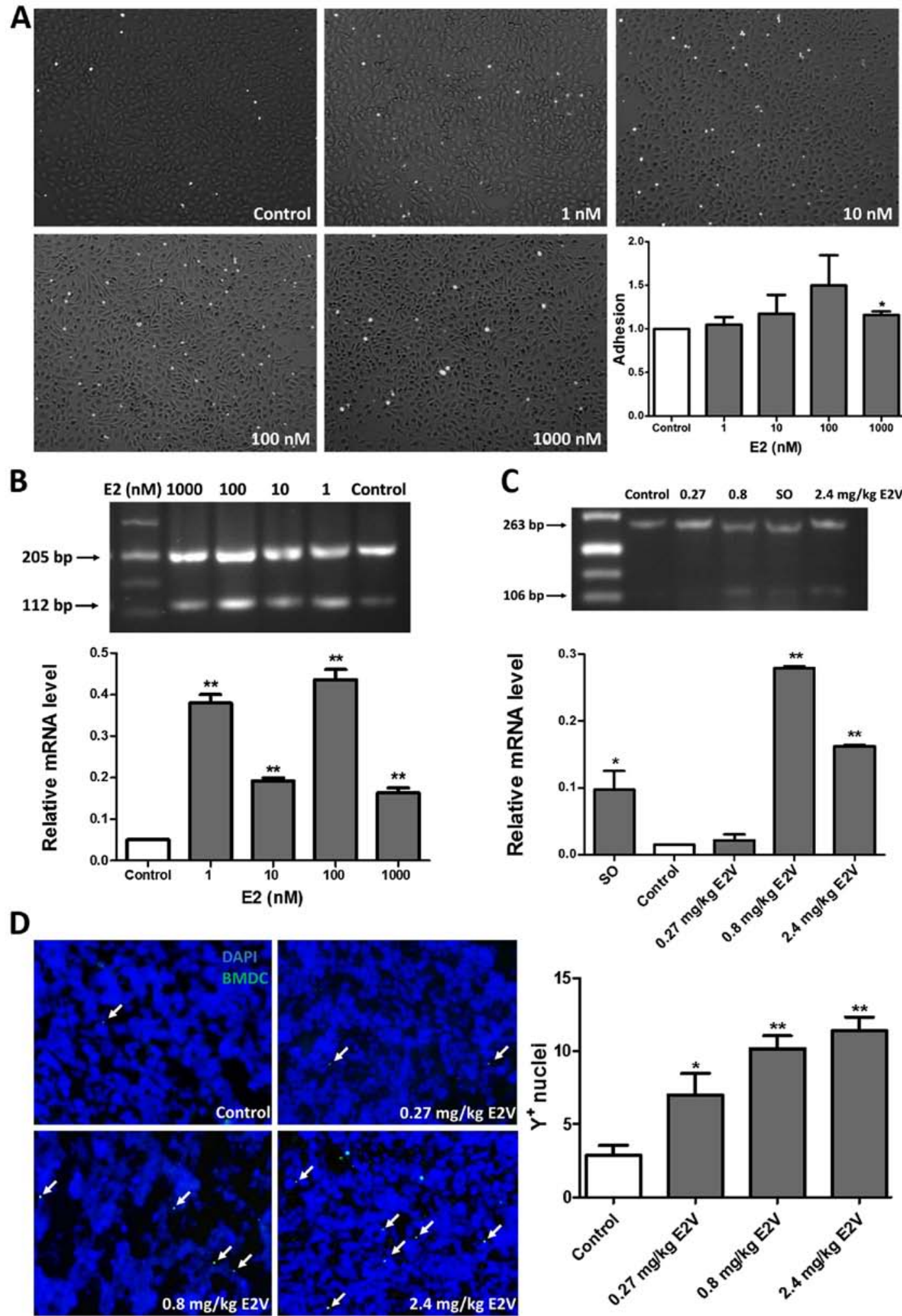

Figure 6. Effects of E2 on recruitment and retention of BMDCs in tumor in vivo and in vitro. (A) E2 treatment of HUVECs increased BMDC adhesion. (B) HUVECs pretreated with E2 for $48 \mathrm{~h}$ induced a significant increase in integrin $\beta_{3}$ mRNA. (C) E2 upregulated the mRNA expression of SDF-1 in tumor tissues. (D) BMDCs in tumor tissues from sex-mismatched BMT mice with male mice as donors. Increased BMDCs were observed with Y-chromosome fluorescence-in-situ-hybridization immunofluorescence staining (green) in female mice. Data are the density of Y-chromosome-positive cells per field of view. Data are mean and SEM values. ${ }^{*} \mathrm{P}<0.05,{ }^{* * *} \mathrm{P}<0.01$ vs. the control. E2, $17 \beta$-estradiol; BMDCs, bone marrow-derived cells; HUVECs, human umbilical vein endothelial cells; BMT, bone marrow transplantation.

significantly at certain concentrations. And E2-treated WBC CM promoted the proliferation of ECs in a nonlinear dose-effect relationship manner. The results suggest that there may be other factors involved in this process, and angiogenesis-related cytokines should be assessed using immunoassay methods such as ELISA, western blotting, IHC and protein 
array. Additionally, even though BMDC CM increased the enhancement of HUVEC proliferation, no obvious difference was found between the BMDC CM and E2-treated BMDC $\mathrm{CM}$ groups. In contrast, both E2 and BMDC CM greatly increased HUVEC migration in comparison with the control, and E2-treated BMDC CM markedly increased the enhancement of HUVEC migration as compared with either the E2 or BMDC CM group. These results reveal that, on the one hand, CM of BMDCs markedly affected the proliferation and migration of ECs, but not the proliferation of 4T1 cells, while on the other hand, CM of BMDCs stimulated with E2 distinctly enhanced the proliferation of 4T1 cells and the function of ECs. Meanwhile, E2 significantly enhanced 4T1 cell proliferation and EC migration.

Moreover, the present study also found that E2 greatly enhanced the BMDC-induced facilitation of HUVEC migration, but not proliferation, with E2 also significantly enhancing 4T1-stimulated EC proliferation. Therefore, E2 enhances tumor growth and angiogenesis by stimulating 4T1 cell proliferation and HUVEC migration directly, and also by stimulating BMDCs to enhance this process indirectly. These results imply that BMDCs may play a key role in mediating E2-induced tumorigenesis and angiogenesis.

The number of BMDCs in 4T1 tumor tissues was observed using a BMT mouse model. These findings confirmed the assumption that E2 increases the recruitment of BMDCs in tumor tissues, which, along with the observation that BMDCs mediate the E2-induced enhancement of tumor cells and EC function in vitro, at least partly elucidated the mechanism via which E2 enhances ER-negative tumor progression and angiogenesis through intensifying the recruitment of BMDCs in local tissues.

BMDCs are mobilized from bone marrow and released into circulating blood, and then are recruited into tumor tissues by passing through the blood vessel endothelium (26). Adhesion molecules such as integrin $\beta_{3}$ (CD61) on the cell surface were found to play an important role in this process (27). Integrin $\beta_{3}$ has the potential to control tumor growth by regulating BMDC adhesion to the vascular endothelium (24). Cytokines and chemokines released by tumor tissues attract BMDCs into tumor tissues (28), of which SDF-1 and its receptor CXCR4 on BMDCs are key partners for BMDC recruitment and trafficking (29). Our previous research demonstrated that almost all of the BMDCs in tumor tissues exhibit positivity for CXCR4. Furthermore, it was reported that $\mathrm{CXCR} 4^{+}, \beta_{3}{ }^{+}$and $\mathrm{CD}_{11} \mathrm{~b}^{+}$myeloid cell homing into tumor tissues accelerates tumor angiogenesis and growth $(24,30,31)$. Thus, $\beta_{3}{ }^{+}, \mathrm{CXCR}^{+}$ and $\mathrm{CD} 11 \mathrm{~b}^{+} \mathrm{BMDCs}$ in circulating blood were analyzed in the present study, with the mRNA transcriptions of $\beta_{3}$ expressed on ECs and SDF-1 in tumor tissues being determined. However, the SO group exhibited a higher level of $\mathrm{CXCR}^{+}$but it did not have altered subpopulations of $\beta_{3}{ }^{+}, \mathrm{CXCR}^{+} \beta_{3}{ }^{+}, \mathrm{CD}_{11 b^{+}}$, and Sca- $1^{+}$BMDCs, which is probably owing to fluctuation of estrogen levels.

Increases in proangiogenic $\beta_{3}{ }^{+}$and/or CXCR4 $4^{+}$BMDCs in blood, the enhancement of $\beta_{3}$ transcription in ECs and SDF-1 mRNA transcriptions in tumor tissues, and the enhancement of adhesion of BMDCs to ECs were found in the present study, which yielded a schematic explanation of how E2 augments BMDCs in tumor tissues. These findings also confirmed previous reports of E2 enhancing ER-negative tumor growth and angiogenesis by influencing the mobilization and recruitment of a proangiogenic population of bone marrow-derived myeloid cells (16).

Previous studies have shown that tumor cells are capable of secreting cytokines so as to stimulate vascular development in response to E2 treatment (32). Our results also indicated that the proliferation of ECs was significantly upregulated by ER-negative 4T1 tumor cells in vitro, and that this process was markedly enhanced by E2. E2 usually binds to classical nuclear ERs that regulate the expression of genes related to the cell cycle and proliferation (33). However, E2 was also found to combine with GPER, as distinguished from the classic ERs, and trigger HIF1 $\alpha$-dependent VEGF expression by activating the GPER, EGFR, ERK, and $c$-fos signaling pathways; the increased VEGF then leads to E2-supported angiogenesis (32). Hence, the above-described experiments revealed enhancement-based interactions involving BMDCs, tumor cells and vascular ECs, and an enhancement role of E2 on this process.

Collectively, the results described herein show that E2 enhances the mobilization of proangiogenic CXCR4 ${ }^{+}, \beta_{3}{ }^{+}$and $\mathrm{CXCR}^{+} \beta_{3}{ }^{+} \mathrm{BMDC}$ from bone marrow into circulating blood. E2 also enhances the transcriptions of integrin $\beta_{3}$ mRNA on ECs and SDF-1 in tumor tissues, thereby facilitating the adhesion of BMDCs to ECs, increasing the recruitment of BMDCs, and resulting in an increased retention of BMDCs in tumor tissues. BMDCs significantly affect the proliferation and migration of ECs, and 4T1 cells markedly enhance the proliferation of ECs, which may improve angiogenesis. These findings also demonstrate that E2 can significantly enhance 4T1 cell proliferation and EC migration directly. Moreover, E2 significantly potentiates the BMDC-induced increase in EC migration and obviously intensifies 4T1-stimulated EC proliferation indirectly. These data represent preliminary evidence of the role of E2 in protumoral processes and may yield novel strategies for use in the development of new drugs.

\section{Acknowledgements}

Not applicable.

\section{Funding}

The present study was supported by the National Natural Science Foundation of China (no. 81071765 and 81372379), the Natural Science Foundation of Shaanxi Province (no. 2016JM8037), and the Research Foundation of The First Affiliated Hospital of Xi'an Jiaotong University (no. 2017MS-09).

\section{Availability of data and materials}

The datasets used during the present study are available from the corresponding author upon reasonable request.

\section{Authors' contributions}

WF and YZ designed the study. YZ, XueL, JC and XZ performed the experiments. QZ, XF and PZ analyzed the data. 
WM, XuaL, FT and KC compiled the figures. YZ and WF wrote the paper. All authors approved the final version and agreed to be accountable for all aspects of the work in ensuring that questions related to the accuracy or integrity of any part of the work are appropriately investigated and resolved.

\section{Ethics approval and consent to participate}

All animal procedures were done in accordance with the protocol approved by the Xi'an Jiaotong University Animal Care and Use Committee.

\section{Patient consent for publication}

Not applicable.

\section{Competing interests}

The authors declare that they have no competing interests.

\section{References}

1. O'Lone R, Frith MC, Karlsson EK and Hansen U: Genomic targets of nuclear estrogen receptors. Mol Endocrinol 18 $1859-1875,2004$

2. Deroo BJ and Korach KS: Estrogen receptors and human disease. J Clin Invest 116: 561-570, 2006.

3. Mauvais-Jarvis F, Clegg DJ and Hevener AL: The role of estrogens in control of energy balance and glucose homeostasis. Endocr Rev 34: 309-338, 2013.

4. Cui J, Shen Y and Li R: Estrogen synthesis and signaling pathways during aging: From periphery to brain. Trends Mol Med 19: 197-209, 2013

5. Nelson ER, Wardell SE and McDonnell DP: The molecular mechanisms underlying the pharmacological actions of estrogens, SERMs and oxysterols: Implications for the treatment and prevention of osteoporosis. Bone 53: 42-50, 2013.

6. Ascenzi P, Bocedi A and Marino M: Structure-function relationship of estrogen receptor alpha and beta: Impact on human health. Mol Aspects Med 27: 299-402, 2006.

7. Hamilton KJ, Arao Y and Korach KS: Estrogen hormone physiology: Reproductive findings from estrogen receptor mutant mice. Reprod Biol 14: 3-8, 2014.

8. Björnström L and Sjöberg M: Mechanisms of estrogen receptor signaling: Convergence of genomic and nongenomic actions on target genes. Mol Endocrinol 19: 833-842, 2005.

9. Santollo J and Daniels D: Multiple estrogen receptor subtypes influence ingestive behavior in female rodents. Physiol Behav 152: 431-437, 2015.

10. Yager JD and Davidson NE: Estrogen carcinogenesis in breast cancer. N Engl J Med 354: 270-282, 2006.

11. McGuire S: World cancer report 2014. Geneva, Switzerland: World health organization, international agency for research on cancer, WHO Press, 2015. Adv Nutr 7: 418-419, 2016.

12. Endogenous Hormones and Breast Cancer Collaborative Group, Key TJ, Appleby PN, Reeves GK, Travis RC, Alberg AJ Barricarte A, Berrino F, Krogh V, Sieri S, et al: Sex hormones and risk of breast cancer in premenopausal women: A collaborative reanalysis of individual participant data from seven prospective studies. Lancet Oncol 14: 1009-1019, 2013.

13. Dunn BK, Wickerham DL and Ford LG: Prevention of hormone-related cancers: Breast cancer. J Clin Oncol 23: $357-367,2005$.

14. Losordo DW and Isner JM: Estrogen and angiogenesis: A review. Arterioscler Thromb Vasc Biol 21: 6-12, 2001.
15. Arnal JF, Fontaine C, Billon-Galés A, Favre J, Laurell H, Lenfant F and Gourdy P: Estrogen receptors and endothelium. Arterioscler Thromb Vasc Biol 30: 1506-1512, 2010.

16. Iyer V, Klebba I, McCready J, Arendt LM, Betancur-Boissel M, Wu MF, Zhang X, Lewis MT and Kuperwasser C: Estrogen promotes ER-negative tumor growth and angiogenesis through mobilization of bone marrow-derived monocytes. Cancer Res 72: 2705-2713, 2012.

17. Feng W, Madajka M, Kerr BA, Mahabeleshwar GH, Whiteheart SW and Byzova TV: A novel role for platelet secretion in angiogenesis: Mediating bone marrow-derived cell mobilization and homing. Blood 117: 3893-3902, 2011.

18. Murdoch C, Muthana M, Coffelt SB and Lewis CE: The role of myeloid cells in the promotion of tumour angiogenesis. Nat Rev Cancer 8: 618-631, 2008.

19. Rizvi AZ, Swain JR, Davies PS, Bailey AS, Decker AD, Willenbring H, Grompe M, Fleming WH and Wong MH: Bone marrow-derived cells fuse with normal and transformed intestinal stem cells. Proc Natl Acad Sci USA 103: 6321-6325, 2006.

20. Ruifrok WP, de Boer RA, Iwakura A, Silver M, Kusano K, Tio RA and Losordo DW: Estradiol-induced, endothelial progenitor cell-mediated neovascularization in male mice with hind-limb ischemia. Vasc Med 14: 29-36, 2009.

21. Hamada H, Kim MK, Iwakura A, Ii M, Thorne T, Qin G, Asai J, Tsutsumi Y, Sekiguchi H, Silver M, et al: Estrogen receptors alpha and beta mediate contribution of bone marrow-derived endothelial progenitor cells to functional recovery after myocardial infarction. Circulation 114: 2261-2270, 2006.

22. Péqueux C, Raymond-Letron I, Blacher S, Boudou F, Adlanmerini M, Fouque MJ, Rochaix P, Noël A, Foidart JM, Krust A, et al: Stromal estrogen receptor-alpha promotes tumor growth by normalizing an increased angiogenesis. Cancer Res 72: 3010-3019, 2012.

23. Ström JO, Theodorsson A, Ingberg E, Isaksson IM and Theodorsson E: Ovariectomy and $17 \beta$-estradiol replacement in rats and mice: A visual demonstration. J Vis Exp 64: e4013, 2012.

24. Feng W, McCabe NP, Mahabeleshwar GH, Somanath PR, Phillips DR and Byzova TV: The angiogenic response is dictated by beta3 integrin on bone marrow-derived cells. J Cell Biol 183: $1145-1157,2008$

25. Houghton J, Stoicov C, Nomura S, Rogers AB, Carlson J, Li H, Cai X, Fox JG, Goldenring JR and Wang TC: Gastric cancer originating from bone marrow-derived cells. Science 306: 1568-1571, 2004.

26. Shaked Y and Voest EE: Bone marrow derived cells in tumor angiogenesis and growth: are they the good, the bad or the evil? Biochim Biophys Acta 1796: 1-4, 2009.

27. Mahabeleshwar GH, Feng W, Phillips DR and Byzova TV: Integrin signaling is critical for pathological angiogenesis. J Exp Med 203: 2495-2507, 2006

28. Melero-Martin JM and Dudley AC: Concise review: Vascular stem cells and tumor angiogenesis. Stem Cells 29: 163-168, 2011.

29. Sainz J and Sata M: CXCR4, a key modulator of vascular progenitor cells. Arterioscler Thromb Vasc Biol 27: 263-265, 2007.

30. Teicher BA and Fricker SP: CXCL12 (SDF-1)/CXCR4 pathway in cancer. Clin Cancer Res 16: 2927-2931, 2010.

31. Ahn GO, Tseng D, Liao CH, Dorie MJ, Czechowicz A and Brown JM: Inhibition of Mac-1 (CD11b/CD18) enhances tumor response to radiation by reducing myeloid cell recruitment. Proc Natl Acad Sci USA 107: 8363-8368, 2010.

32. De Francesco EM, Pellegrino M, Santolla MF, Lappano R, Ricchio E, Abonante S and Maggiolini M: GPER mediates activation of HIF1 $\alpha /$ VEGF signaling by estrogens. Cancer Res 74: 4053-4064, 2014.

33. Heldring N, Pike A, Andersson S, Matthews J, Cheng G, Hartman J, Tujague M, Ström A, Treuter E, Warner M and Gustafsson JA: Estrogen receptors: How do they signal and what are their targets. Physiol Rev 87: 905-931, 2007. 